\title{
Combination of histoculture drug response assay and qPCR as an effective method to screen biomarkers for personalized chemotherapy in esophageal cancer
}

\author{
BIN WEI $^{1 *}$, JIRU WANG $^{1 *}$, XIAOHUI ZHANG ${ }^{2 *}$, ZHAOYE QIAN $^{1}$, JINGJING WU $^{3}$, \\ YUAN SUN $^{1}$, QIN HAN ${ }^{1}$, LI WAN ${ }^{1}$, JING ZHU $^{1}$, YONG GAO ${ }^{1}$ and XIAOFEI CHEN ${ }^{1}$
}

\begin{abstract}
${ }^{1}$ Department of Medical Oncology, Huai'an First People's Hospital, Nanjing Medical University, Huai'an, Jiangsu 223300;
${ }^{2}$ Department of Medical Oncology, The Second Affiliated Hospital of Nanjing Medical University, Nanjing, Jiangsu 210011;

${ }^{3}$ Department of Hematology, Huai'an First People's Hospital, Nanjing Medical University, Huai'an, Jiangsu 223300, P.R. China
\end{abstract}

Received April 12,2016; Accepted June 27, 2017

DOI: $10.3892 / 01.2017 .7069$

\begin{abstract}
Personalized chemotherapy with the use of biomarkers helps to maximize clinical efficiency. Therefore, the present study aimed to identify a potential method for identifying biomarkers in esophageal cancer. A total of 49 freshly resected tumor tissues and 72 paraffin-embedded specimens from patients with esophageal cancer were obtained. mRNA expression levels of ERCC1, BRCA1, TUBB3, FBW7, RRM1, MDM2, TS and TOP1 were measured quantitative reverse transcription polymerase chain reaction (RT-qPCR). In vitro chemosensitivity to cisplatin, docetaxel, gemcitabine, etoposide, fluorouracil and irinotecan were tested using histoculture drug response assay (HDRA). BRCA1 mRNA levels were positively correlated with resistance to cisplatin $(\mathrm{P}=0.027)$ and sensitivity to docetaxel $(\mathrm{P}=0.002)$. TS mRNA levels were inversely correlated with fluorouracil sensitivity $(\mathrm{P}=0.044)$, and TOP1 mRNA expression was positively correlated with irinotecan sensitivity $(\mathrm{P}=0.008)$. In addition, high BRCA1 mRNA levels correlated with decreased median overall survival $(\mathrm{mOS} ; \mathrm{P}<0.001)$ and response rate $(\mathrm{RR} ; \mathrm{P}=0.002)$ in cisplatin-fluorouracil chemotherapy group and also correlated with increased mOS $(\mathrm{P}<0.001)$ and $\mathrm{RR}(\mathrm{P}=0.023)$ in docetaxel-fluorouracil chemotherapy group. Overall, these results suggested that HDRA combined with RT-qPCR may
\end{abstract}

Correspondence to: Dr Yong Gao or Dr Xiaofei Chen, Department of Medical Oncology, Huai'an First People's Hospital, Nanjing Medical University, 6 Beijing West Road, Huai'an, Jiangsu 223300, P.R. China

E-mail: hayy_gy@163.com

E-mail: hayycxf@163.com

*Contributed equally

Key words: histoculture drug response assay, quantitative polymerase chain reaction, personalized chemotherapy, biomarker, esophageal cancer serve as an effective method for screening biomarkers in personalized chemotherapy for esophageal cancer.

\section{Introduction}

Esophageal cancer is one of the leading causes of cancer-associated mortalities in China $(1,2)$. Chemotherapy has been considered an essential method to treat esophageal cancer. However, the efficacy of the chemotherapeutic agents has been limited with rates between 11 and 35\% (3-5). Furthermore, to date, there is no 'gold standard' chemotherapy for esophageal cancer. With the development of pharmacogenomics and pharmacogenetics, tumor heterogeneity is considered to be a significant factor that is responsible for the failure of conventional chemotherapeutics (6,7). In predictive biomarker studies, a number of genes have been reported to predict response to chemotherapy for solid tumors, including excision repair cross-complementation group 1 (ERCC1), breast cancer type 1 gene (BRCA1) or ADP ribosylation factor like GTPase 6 interacting protein 5 (JWA) for cisplatin (8), BRCA1, tubulin $\beta-3$ class III (TUBB3) or F-box and WD repeat domain containing 7 (FBW7) for docetaxel (9-11), thymidylate synthetase (TS) for fluorouracil (12), and ribonucleotide reductase catalytic subunit M1 (RRM1) for gemcitabine (13), murine double minute 2 (MDM2) for etoposide (14) and DNA topoisomerase 1 (TOP1) for irinotecan (15). Additionally, a few of the genes, including BRCA1, JWA and TS, have been validated for their clinical value in esophageal cancer $(8,12)$. However, the clinical value of the aforementioned biomarkers for chemotherapeutic agents such as irinotecan (16), gemcitabine (5) and etoposide (17), which are not commonly used but exhibit moderate activity for treating esophageal cancer, remains unclear. Therefore, it is important to investigate practical methods, which can be used to screen and identify appropriate biomarkers for personalized therapy of esophageal cancer with chemotherapeutic agents of which there are limited clinical application data available.

The histoculture drug response assay (HDRA) is one of a number of in vitro tests for chemosensitivity, which allows the characteristics of the three-dimensional tissue structure to be maintained (18). HDRA has the advantage of being able 
to maintain three-dimensional tissue structure and may be able to more accurately mimic the in vivo response compared with a cell culture model $(18,19)$. The clinical reliability and utility of HDRA have been examined in several clinical studies for various solid tumors, including oral squamous cell carcinoma, head and neck, gastric, colorectal and ovarian cancer (20-22). Furthermore, HDRA has gradually been applied to identify candidate genes or gene sets with the capacity to predict efficiency of chemotherapeutic and targeted agents. Therefore, in the present study, HDRA was employed to evaluate the sensitivity of chemotherapeutic agents (cisplatin, docetaxel, gemcitabine, etoposide, fluorouracil and irinotecan) in tumor tissues, and the quantitative reverse transcription polymerase chain reaction method was performed to detect the mRNA expression of ERCC1, BRCA1, TUBB3, FBW7, RRM1, MDM2, TS and TOP1. Additionally, the present study verified the predictive value of a potential biomarker in patients with advanced esophageal cancer.

\section{Patients and methods}

Patients and sample collection. All patients and relevant clinical data were obtained from the Huai'an First People's Hospital, Nanjing Medical University (Huai'an, China) from May 2012 to June 2013. The median age was 62, and the majority of patients were male. Written informed consent for the use of tissue specimens was obtained from all patients, and the protocols for the present study were approved by the Ethics Committee of Huai'an First People's Hospital, Nanjing Medical University.

The surgically resected tumor specimens were obtained from 49 patients. Each specimen was divided into three parts. One part of the specimens was kept in $4^{\circ} \mathrm{C}$ Hanks' balanced salt solution with $1 \%$ penicillin/streptomycin, and HDRA was employed to measure inhibition rates of chemotherapeutic agents in vitro within $15 \mathrm{~min}$. Another part of the specimen was fixed by $10 \%$ formalin for $24 \mathrm{~h}$ at room temperature and embedded with paraffin for pathological observation. The rest of the tissue was stored in $-80^{\circ} \mathrm{C}$ for further detection of gene expression.

The paraffin-embedded tumor materials were collected from 72 cases with advanced esophageal cancer that received cisplatin-fluorouracil (cisplatin $25 \mathrm{mg} / \mathrm{m}^{2}$ on day 1-3; fluorouracil $500 \mathrm{mg} / \mathrm{m}^{2}$ on day 1-5) or docetaxel-fluorouracil (docetaxel $60-75 \mathrm{mg} / \mathrm{m}^{2}$; fluorouracil $500 \mathrm{mg} / \mathrm{m}^{2}$ on day $1-5$ ) chemotherapy. Chemotherapy was repeated every 3-4 weeks for a maximum of six cycles unless patients had disease progression or in unsupportable adverse reactions.

$H D R A$. HDRA procedures were performed as previously described by Furukawa et al (18). Cancerous portions of specimens were washed three times with Hank's balanced salt solution and divided into $\sim 10 \mathrm{mg}$ pieces. Then, the tissue fragments were placed on prepared collagen sponge surfaces (Health Design, Rochester, NY, USA) in 24-well plates and incubated for 6 days in RPMI 1640 medium (Gibco; Thermo Fisher Scientific, Inc., Waltham, MA, USA) supplemented with $20 \%$ fetal bovine serum (Gibco; Thermo Fisher Scientific, Inc.) at $37^{\circ} \mathrm{C}$ in $5 \% \mathrm{CO}_{2}$. The concentration of each agent was determined by a preliminary experiment with $25 \%$ growth inhibition ( $\mathrm{IC}_{25}$ value) as follows: $100 \mu \mathrm{g} / \mathrm{ml}$ for cisplatin, $30 \mu \mathrm{g} / \mathrm{ml}$ for docetaxel (Jiangsu Hengrui Medicine Co., Ltd., Nanjing, China), $10 \mu \mathrm{g} / \mathrm{ml}$ for fluorouracil, $30 \mu \mathrm{g} / \mathrm{ml}$ for gemcitabine, $10 \mu \mathrm{g} / \mathrm{ml}$ for etoposide and $10 \mu \mathrm{g} / \mathrm{ml}$ for irinotecan (Jiangsu Haosen Medicine Company, Nanjing, China).

Following histoculture, $100 \mu \mathrm{l}$ Hank's balanced salt solution containing $0.1 \mathrm{mg} / \mathrm{ml}$ type I collagenase (Sigma-Aldrich; Merck KGaA, Darmstadt, Germany) and $100 \mu \mathrm{l}$ MTT solution ( $2 \mathrm{mg} / \mathrm{ml}$ ) were added to each well. The plates were incubated at $37^{\circ} \mathrm{C}$ for another $24 \mathrm{~h}$. Following extraction with dimethyl sulfoxide, the absorbance of the solution in each well was measured with microplate reader at $540 \mathrm{~nm}$. Absorbance per gram of cultured tumor tissue was calculated from the mean absorbance from 8 parallel culture wells, and the weight of tumor tissue was determined prior to culture. The inhibition rate (IR) was calculated using the following formula: $\mathrm{IR}=(1-\mathrm{T} / \mathrm{C}) \mathrm{x} 100 \%$, where $\mathrm{T}$ is the mean absorbance of the treated tumor/weight, and $\mathrm{C}$ is the mean absorbance of the control tumor/weight.

Quantitative reverse transcription polymerase chain reaction $(R T-q P C R)$. Frozen tissues ( $10 \mathrm{mg} /$ per sample) were grinded in liquid nitrogen, and the total RNA was extracted by using the TRIzol reagent (Invitrogen; Thermo Fisher Scientific, Inc.). For paraffin-embedded tumor specimens, micro-dissection was performed to ensure serial sections of $7 \mathrm{~mm}$ in thickness with $>80 \%$ of tumor cells. The pellet of micro-dissected cells was resuspended in Trizol reagent supplemented with proteinase $\mathrm{K}$ to extract RNA. Then RNA was reverse-transcribed with FastQuant RT kit (Tiangen Biotech Co., Ltd., Beijing, China). Each sample was detected in triplicate with RNase-free water, and commercial RNA as negative and positive control. Template cDNA was amplified with specific primers for different genes with the SuperReal PreMix Plus (Tiangen Biotech Co., Ltd., Beijing, China) by using the Real-Time PCR Detection system (Roche Applied Science Madison, WI, USA). The sequences of the primers are provided in Table I. Relative gene expression quantification was calculated using the $\mathrm{Cq}$ method. Final values were determined by the formula $2^{-\Delta \Delta \mathrm{Cq}}$ and were analyzed with the Stratagene analysis software (version Mx3000P; Agilent Technologies, Inc., Santa Clara, CA, USA).

Statistical analysis. The Mann-Whitney U and Kruskal-Wallis tests were used to analyze the association between inhibition rates of agents or gene expression levels and clinical characteristics. The mean value was employed as the cutoff point of gene levels to divide the patients into low or high expression groups. The Mann-Whitney U test was used to compare the inhibition rates between the two groups. Clinical response was evaluated according to the Response Evaluation Criteria in Solid Tumors (23). Overall survival (OS) was calculated from the date of diagnosis to the date of last follow-up or mortality from any cause. The distributions of OS were analyzed using Kaplan-Meier method and compared with the two-sided log-rank test. $\mathrm{P}<0.05$ was considered to indicate a statistically significant difference. Statistical analyses were performed by SPSS 19.0 software (IBM Corp., Armonk, NY, USA). 
Table I. Primer sequences used for gene analysis.

\begin{tabular}{lll}
\hline Genes & \multicolumn{1}{c}{ Forward primer $\left(5^{\prime}-3^{\prime}\right)$} & \multicolumn{1}{c}{ Reverse primer $\left(5^{\prime}-3^{\prime}\right)$} \\
\hline ERCC1 & ACCCCTCGACGAGGATGA & GATGGC ATATTCGGCGTAGGT \\
BRCA1 & TCCCATCTGTCTGGAGTTGA & GCCCTTTCTTCTGGTTGAGA \\
TUBB3 & GCAGTCGCAGTTTTCACACTC & GCAGTCGCAGTTTTCACACTC \\
FBXW7 & GGCCAAAATGATTCCCAGCAA & ACTGGAGTTCGTGACACTGTTA \\
RRM1 & AGCAGCCAAAGTATCTAGTTCCA & AGCAGCCAAAGTATCTAGTTCCA \\
MDM2 & TCGTCGGGTGAGGGTACTG & AACCACTTCTTGGAACCAGGT \\
TOP1 & CTTCAGCGAGAACCCAGACC & TCCAGCCCAACCCCTAAAGAC \\
$\beta$-actin & GAGAGCTGTAGCCCTGTACTTCATC & CAGTGTCCGCTGTTTCTCCTT \\
\hline
\end{tabular}

ERCC1, excision repair cross-complementation group 1; BRCA1, breast cancer type 1 gene; TUBB3, tubulin $\beta$-3 class III; FBXW7, F-box and WD repeat domain containing 7; RRM1, ribonucleotide reductase catalytic subunit M1; MDM2, murine double minute 2; TS, thymidylate synthetase; TOP1, DNA topoisomerase 1.

Table II. Clinical characteristics of patients with esophageal cancer.

\begin{tabular}{|c|c|c|c|c|}
\hline \multirow[b]{2}{*}{ Clinical values } & \multirow[b]{2}{*}{ HDRA cohort } & \multicolumn{3}{|c|}{ Clinical cohort } \\
\hline & & Total & Cisplatin-fluorouracil & Docetaxel-fluorouracil \\
\hline \multicolumn{5}{|l|}{ Sex, n (\%) } \\
\hline Male & $31(63.3)$ & $45(62.5)$ & $21(61.7)$ & $24(63.2)$ \\
\hline Female & $18(36.7)$ & $27(37.5)$ & $13(38.2)$ & $14(36.8)$ \\
\hline \multicolumn{5}{|l|}{ Age, n (\%) } \\
\hline$\leq 62$ & $27(55.1)$ & $40(55.6)$ & $21(61.7)$ & $19(50.0)$ \\
\hline$>62$ & $22(44.9)$ & $32(44.4)$ & $13(38.2)$ & $19(50.0)$ \\
\hline \multicolumn{5}{|l|}{ Tumor site, n (\%) } \\
\hline Upper & $5(10.2)$ & $7(9.7)$ & $4(11.8)$ & $3(7.9)$ \\
\hline Middle & $29(59.2)$ & $52(72.2)$ & $23(67.6)$ & $29(76.3)$ \\
\hline Lower & $15(30.6)$ & $13(18.1)$ & $7(20.6)$ & $6(15.7)$ \\
\hline \multicolumn{5}{|l|}{ Histological grade, n (\%) } \\
\hline 1 & $10(20.4)$ & $3(4.1)$ & $2(5.9)$ & $1(2.6)$ \\
\hline 2 & $39(79.6)$ & $49(68.1)$ & $25(73.5)$ & $24(63.1)$ \\
\hline 3 & $0(0.0)$ & $20(27.8)$ & $7(20.5)$ & $13(34.2)$ \\
\hline \multicolumn{5}{|l|}{ Stage, n (\%) } \\
\hline II & $27(55.1)$ & $0(0.0)$ & $0(0.0)$ & $0(0.0)$ \\
\hline III & $22(44.9)$ & $4(5.6)$ & $2(5.9)$ & $1(2.6)$ \\
\hline IV & $0(0.0)$ & $68(94.4)$ & $32(94.1)$ & $37(97.4)$ \\
\hline \multicolumn{5}{|l|}{ Response rate, n (\%) } \\
\hline $\mathrm{CR}+\mathrm{PR}$ & & & $13(38.2)$ & $14(36.8)$ \\
\hline $\mathrm{SD}+\mathrm{PD}$ & & & $21(61.8)$ & $24(63.2)$ \\
\hline mOS (months, $95 \% \mathrm{CI}$ ) & & & $13.2(11.3-17.0)$ & $10.3(9.5-14.3)$ \\
\hline
\end{tabular}

$\mathrm{CI}$, confidence interval; HDRA, histoculture drug response assay; CR, complete response; PR, partial response; SD, stable disease; PD, progressive disease; mOS, median overall survival.

\section{Results}

Patient characteristics. Characteristics of all patients are shown in Table II. In the HDRA cohort, all patients were in stages II-III (24) at the time of diagnosis. In the clinical cohort, the patients were restricted to stages III-IV. In the clinical cohort, 34 patients treated with cisplatin-fluorouracil-based chemotherapy with response rate (RR) of $38.2 \%$ 
Table III. Association between inhibition rates of chemotherapy agents and clinical characteristics.

Inhibition rates $(\%)$ of chemotherapy agents (mean and $95 \% \mathrm{CI})^{\mathrm{a}}$

\begin{tabular}{|c|c|c|c|c|c|c|}
\hline Clinical values & Cisplatin & Docetaxel & Gemcitabine & Etoposide & Fluorouracil & Irinotecan \\
\hline \multicolumn{7}{|l|}{ Sex } \\
\hline Male & 39.1 (31.2-46.9) & $21.3(15.9-26.7)$ & $18.4(13.7-23.0)$ & $20.1(14.9-25.1)$ & $17.5(13.9-21.2)$ & $20.5(14.7-26.3)$ \\
\hline Female & $45.9(36.3-55.5)$ & $14.9(9.6-20.3)$ & $28.7(19.3-38.0)$ & $19.7(12.9-26.5)$ & $22.9(14.6-31.3)$ & $22.4(14.2-30.5)$ \\
\hline \multicolumn{7}{|l|}{ Age } \\
\hline$\leq 62$ & $42.0(34.5-49.5)$ & $21.3(16.5-26.2)$ & $21.5(15.5-27.4)$ & $22.3(16.6-28.1)$ & $21.4(15.9-26.8)$ & $25.1(19.0-31.2)$ \\
\hline$>62$ & $41.1(30.8-51.4)$ & $16.1(9.4-22.7)$ & $23.0(15.4-30.6)$ & $16.9(11.6-22.3)$ & $17.3(11.9-22.6)$ & $16.4(9.6-23.2)$ \\
\hline \multicolumn{7}{|l|}{ Tumor site } \\
\hline Upper & $39.0(12.3-65.8)$ & $33.8(12.4-55.3)$ & $30.4(0.0-61.2)$ & $26.8(12.1-41.4)$ & $29.1(14.8-43.3)$ & $22.9(5.1-50.9)$ \\
\hline Middle & $43.9(35.8-52.2)$ & $15.4(11.2-19.5)$ & $21.4(16.7-26.1)$ & $18.9(13.6-24.3)$ & $19.5(14.2-24.7)$ & $20.0(15.0-25.0)$ \\
\hline Lower & $37.8(27.0-48.7)$ & $21.0(13.1-28.9)$ & $20.9(10.6-31.3)$ & $19.5(12.0-29.9)$ & $16.4(10.4-22.5)$ & $23.0(12.5-33.4)$ \\
\hline \multicolumn{7}{|c|}{ Histological grade } \\
\hline 1 & $39.7(20.1-59.3)$ & $22.6(14.6-30.7)$ & $21.7(8.9-34.5)$ & $27.4(15.4-39.4)$ & $22.2(11.1-33.4)$ & $26.2(10.8-41.5)$ \\
\hline 2 & $42.1(35.9-48.3)$ & $18.1(13.5-22.6)$ & $22.3(17.2-27.3)$ & $18.0(14.0-21.9)$ & $18.8(14.8-22.8)$ & $19.9(15.4-23.5)$ \\
\hline \multicolumn{7}{|l|}{ Stage } \\
\hline II & $44.4(35.9-52.9)$ & $17.2(11.9-22.4)$ & $24.2(16.7-31.6)$ & $21.4(15.8-26.9)$ & $22.0(16.1-27.9)$ & $21.5(13.6-29.4)$ \\
\hline III & $38.1(29.4-46.8)$ & $21.2(15.0-27.4)$ & $19.7(14.8-24.6)$ & $18.1(12.3-23.9)$ & $16.4(12.3-20.6)$ & $20.9(17.0-24.7)$ \\
\hline
\end{tabular}

${ }^{\mathrm{a} A l l} \mathrm{P}>0.05$. CI, confidence interval.

Table IV. Association between gene expression and clinical characteristics.

\begin{tabular}{|c|c|c|c|c|c|c|c|c|}
\hline \multirow[b]{2}{*}{ Clinical values } & \multicolumn{8}{|c|}{ Genes mRNA expression levels (mean \pm standard error) ${ }^{\mathrm{a}}$} \\
\hline & ERCC1 & BRCA1 & TUBB3 & FBW7 & RRM1 & MDM2 & $\mathrm{TS}$ & TOP1 \\
\hline \multicolumn{9}{|l|}{ Sex } \\
\hline Male & $4.4 \pm 0.5$ & $1.1 \pm 0.1$ & $1.0 \pm 0.2$ & $5.0 \pm 0.9$ & $1.7 \pm 0.2$ & $5.5 \pm 0.9$ & $1.7 \pm 0.4$ & $3.4 \pm 0.9$ \\
\hline Female & $5.2 \pm 1.0$ & $1.4 \pm 0.3$ & $1.3 \pm 0.4$ & $9.0 \pm 3.8$ & $1.9 \pm 0.5$ & $7.1 \pm 1.4$ & $1.2 \pm 0.2$ & $4.0 \pm 0.8$ \\
\hline \multicolumn{9}{|l|}{ Age } \\
\hline$\leq 62$ & $4.5 \pm 0.6$ & $1.0 \pm 0.1$ & $0.7 \pm 0.1$ & $7.0 \pm 2.6$ & $1.7 \pm 0.2$ & $5.2 \pm 0.9$ & $1.5 \pm 0.4$ & $3.6 \pm 1.0$ \\
\hline$>62$ & $4.2 \pm 0.8$ & $1.5 \pm 0.3$ & $1.5 \pm 0.4$ & $5.9 \pm 1.0$ & $1.9 \pm 0.4$ & $7.2 \pm 1.3$ & $1.6 \pm 0.3$ & $3.7 \pm 0.7$ \\
\hline \multicolumn{9}{|l|}{ Tumor site } \\
\hline Upper & $3.5 \pm 0.4$ & $1.0 \pm 0.2$ & $0.6 \pm 0.3$ & $2.6 \pm 1.7$ & $1.8 \pm 0.5$ & $4.0 \pm 1.6$ & $2.6 \pm 1.7$ & $1.9 \pm 0.7$ \\
\hline Middle & $5.3 \pm 0.7$ & $1.4 \pm 0.2$ & $1.2 \pm 0.3$ & $8.0 \pm 2.5$ & $2.0 \pm 0.3$ & $7.4 \pm 1.1$ & $1.6 \pm 0.3$ & $4.3 \pm 1.0$ \\
\hline Lower & $4.0 \pm 0.8$ & $1.0 \pm 0.1$ & $0.9 \pm 0.3$ & $4.9 \pm 0.8$ & $1.4 \pm 0.3$ & $4.2 \pm 0.9$ & $1.1 \pm 0.3$ & $2.9 \pm 0.6$ \\
\hline \multicolumn{9}{|c|}{ Histological grade } \\
\hline 1 & $4.8 \pm 1.4$ & $1.2 \pm 0.2$ & $0.8 \pm 0.3$ & $4.4 \pm 1.1$ & $1.3 \pm 0.3$ & $4.4 \pm 1.1$ & $1.3 \pm 1.1$ & $3.4 \pm 1.3$ \\
\hline 2 & $4.7 \pm 0.5$ & $1.2 \pm 0.2$ & $1.1 \pm 1.2$ & $7.0 \pm 1.9$ & $1.9 \pm 0.3$ & $6.5 \pm 0.9$ & $1.6 \pm 0.3$ & $3.7 \pm 0.7$ \\
\hline \multicolumn{9}{|l|}{ Stage } \\
\hline II & $5.1 \pm 0.7$ & $1.3 \pm 0.2$ & $1.1 \pm 0.3$ & $6.1 \pm 1.1$ & $1.9 \pm 0.3$ & $7.2 \pm 1.2$ & $1.5 \pm 0.2$ & $4.1 \pm 1.0$ \\
\hline III & $4.2 \pm 0.7$ & $1.1 \pm 0.1$ & $1.0 \pm 0.3$ & $7.0 \pm 3.1$ & $1.6 \pm 0.2$ & $4.7 \pm 0.9$ & $1.6 \pm 0.5$ & $3.1 \pm 0.6$ \\
\hline
\end{tabular}

aAll P>0.05. ERCC1, excision repair cross-complementation group 1; BRCA1, breast cancer type 1 gene; TUBB3, tubulin $\beta-3$ class III; FBXW7, F-box and WD repeat domain containing 7; RRM1, ribonucleotide reductase catalytic subunit M1; MDM2, murine double minute 2; TS, thymidylate synthetase; TOP1, DNA topoisomerase 1.

and median OS (mOS) of 13.2 [95\% confidence interval $(\mathrm{CI})$, 11.3-17.0] months, while the other 38 patients received the docetaxel-fluorouracil-based chemotherapy with RR of $36.8 \%$ and mOS of 10.3 (95\% CI, 9.5-14.3) months. 


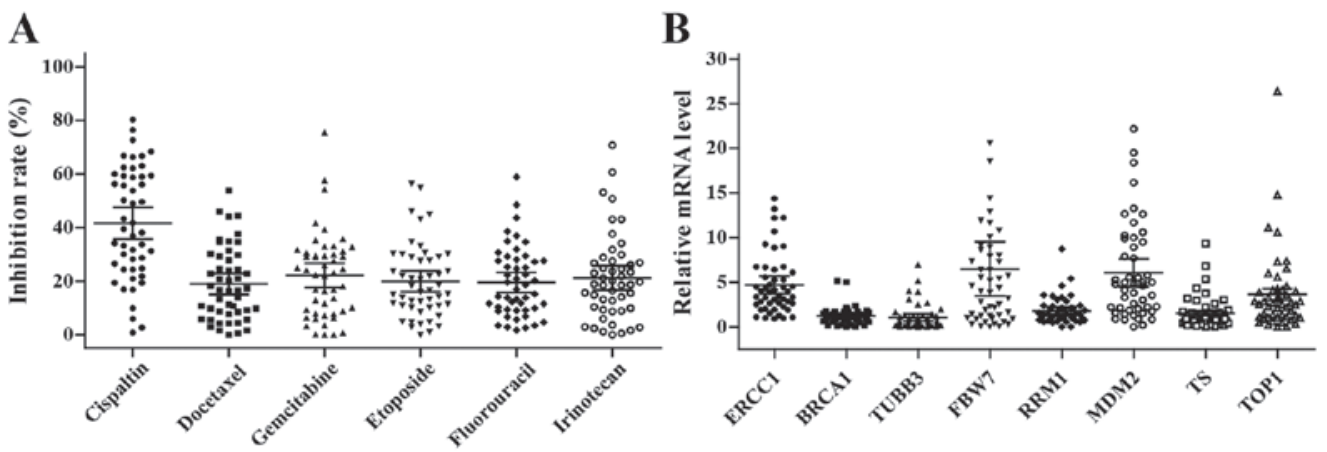

Figure 1. Chemosensitivity and gene expression levels in esophageal cancer tissues. (A) In vitro chemosensitivity to cisplatin, docetaxel, gemcitabine, etoposide, fluorouracil and irinotecan were tested using histoculture drug response assay. (B) The levels of ERCC1, BRCA1, TUBB3, FBW7, RRM1, MDM2, TS and TOP1 mRNA expression in tumor tissues were analyzed by quantitative PCR. The bars indicate the mean and 95\% confidence interval. ERCC1, excision repair cross-complementation group 1; BRCA1, breast cancer type 1 gene; TUBB3, tubulin $\beta$-3 class III; FBXW7, F-box and WD repeat domain containing 7 ; RRM1, ribonucleotide reductase catalytic subunit M1; MDM2, murine double minute 2; TS, thymidylate synthetase; TOP1, DNA topoisomerase 1.

A

D

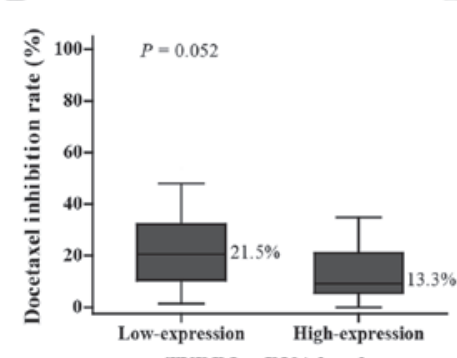

TUBB3 mRNA levels

G

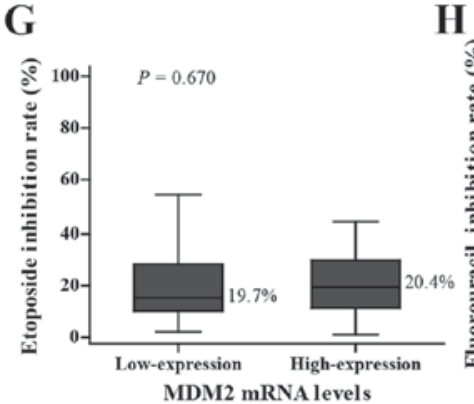

B

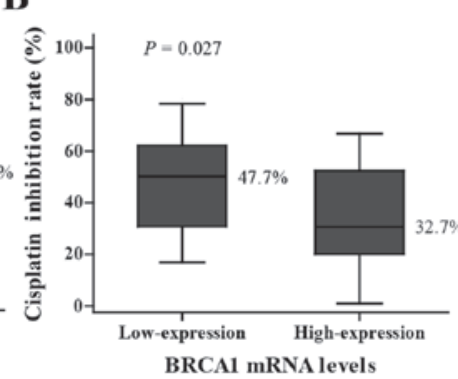

$\mathbf{E}$

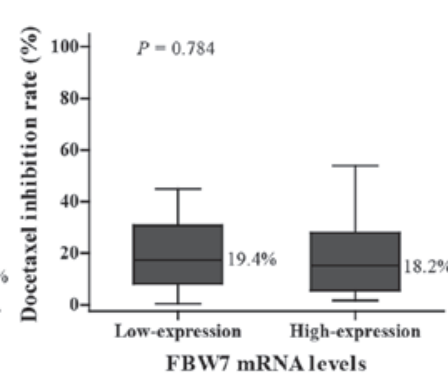

$\mathbf{H}$

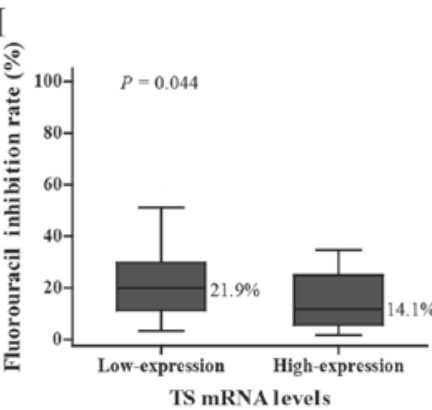

C

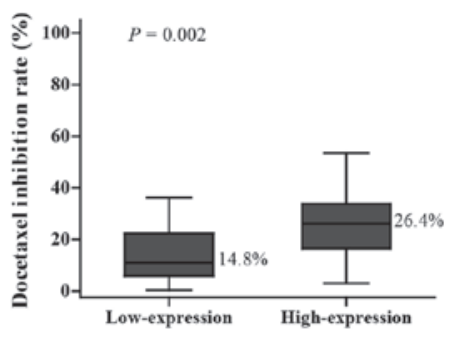

BRCA1 mRNA levels

F

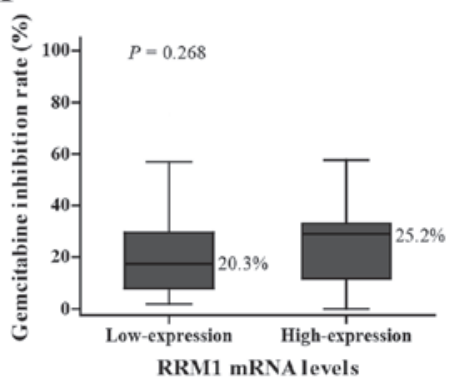

I

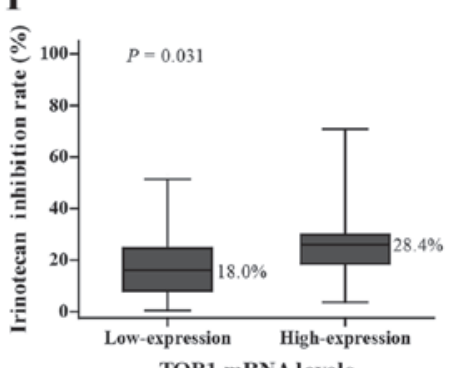

Figure 2. Association between gene expression levels and chemosensitivity. The 49 samples were divided into two subgroups by mean gene expression. The box plots indicate the inhibition rates of chemotherapeutic agents (A) ERCC1 and cisplatin; (B) BRCA1 and cisplatin; (C) BRCA1 and docetaxel; (D) TUBB3 and docetaxel; (E) FBW7 and docetaxel; (F) RRM1 and gemcitabine; (G) MDM2 and etoposide; (H) TS and fluorouracil; and (I) TOP1 and irinotecan. The bars indicate the mean, 5th and 95th percentile. ERCC1, excision repair cross-complementation group 1; BRCA1, breast cancer type 1 gene; TUBB3, tubulin $\beta-3$ class III; FBW7, F-box and WD repeat domain containing 7; RRM1, ribonucleotide reductase catalytic subunit M1; MDM2, murine double minute 2; TS, thymidylate synthetase; TOP1, DNA topoisomerase 1.

Inhibition rates of chemotherapeutic agents. The ability of 6 chemotherapeutic agents to inhibit the growth of 49 tumor specimens was successfully tested using HDRA. Not only did the spectrum of sensitive agent vary between individual 
Table V. Outcomes in different chemotherapy groups stratified by BRCA1 expression.

\begin{tabular}{|c|c|c|c|c|c|c|c|}
\hline \multirow[b]{2}{*}{ Treatments } & \multirow[b]{2}{*}{ BRCA1 } & \multirow[b]{2}{*}{ No. } & \multicolumn{2}{|l|}{$\mathrm{mOS}$} & \multicolumn{3}{|c|}{$\mathrm{RR}, \mathrm{n}(\%)$} \\
\hline & & & Median $(95 \% \mathrm{CI})$ & P-value & $\mathrm{CR}+\mathrm{PR}$ & $\mathrm{SD}+\mathrm{PD}$ & P-value \\
\hline \multirow[t]{2}{*}{ Cisplatin/fluorouracil } & Low & 21 & $16.5(14.6-18.4)$ & & 38.1 & 61.9 & \\
\hline & High & 13 & $6.5(5.4-7.6)$ & $<0.001$ & 7.7 & 92.3 & 0.002 \\
\hline \multirow[t]{2}{*}{ Docetaxel/fluorouracil } & Low & 24 & $6.8(5.7-7.9)$ & & 20.8 & 79.2 & \\
\hline & High & 14 & $22.0(14.0-26.8)$ & $<0.001$ & 57.1 & 42.9 & 0.023 \\
\hline
\end{tabular}

BRCA1, breast cancer type 1 susceptibility protein; CI, confidence interval; mOS, median overall survival; RR, response rate; CR, complete response; $\mathrm{PR}$, partial response; $\mathrm{SD}$, stable disease; $\mathrm{PD}$, progressive disease.
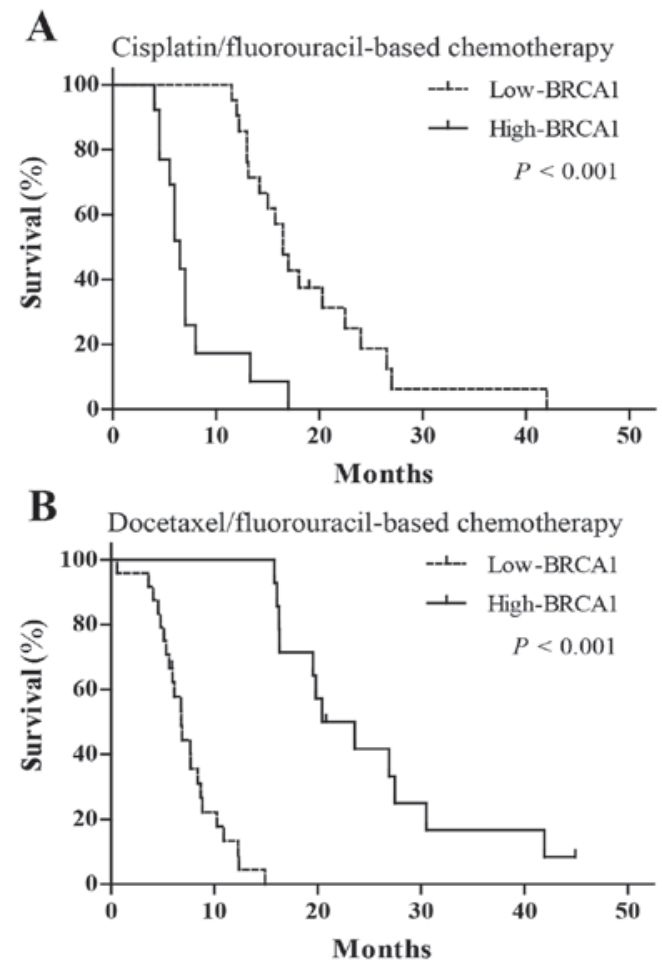

Figure 3. Median overall survival according to BRCA1 mRNA expression in different treatment groups. (A) Cisplatin-fluorouracil chemotherapy group. (B) Docetaxel-fluorouracil chemotherapy group. BRCA1, breast cancer type 1 gene; mRNA, messenger RNA.

patients, but the inhibition rate of each agent also varied and ranged widely. The average inhibition rates were as follows: Cisplatin, 37.6\% (95\% CI, 35.6-47.5\%); docetaxel, $20.0 \%$ (95\% CI, 15.1-22.9\%); gemcitabine, 22.2\% (95\% CI, 16.0-23.8\%); etoposide, $19.9 \%$ (95\% CI, 16.0-23.8\%); fluorouracil, $19.5 \%$ (95\% CI, 15.8-23.2\%); and irinotecan, $21.2 \%$ (95\% CI, $16.7-25.7 \%$ ) (Fig. 1A). However, there was no significant association between inhibition rates and clinical characteristics (all $\mathrm{P}>0.05$, Table III).

Gene expression levels. The mRNA expression levels of 8 genes were detected in all tumor tissues, with mean levels of 4.7 (95\% CI, 3.7-5.7) for ERCC1; 1.2 (95\% CI, 0.9-1.5) for BRCA1; 1.1 (95\% CI, 0.7-1.5) for TUBB3; 6.5 (95\% CI, 3.5-9.5) for FBW7; 1.8 (95\% CI, 1.3-2.2) for RRM1; 6.1
(95\% CI, 4.5-7.6) for MDM2; 1.5 (95\% CI, 1.0-2.1) for TS; and 3.6 (95\% CI, 2.4-4.9) for TOP1 (Fig. 1B). No association between clinical characteristics and gene expression was identified (all P>0.05, Table IV).

Association between gene expression and chemosensitivity. The inhibition rates of various chemotherapeutic agents are indicated in Fig. 2. The inhibition rate of cisplatin in the group with low BRCA1 mRNA expression was higher compared with the group with high expression (44.7 vs. $32.7 \%$; $\mathrm{P}=0.027$; low expression vs. high expression group; Fig. 2B), while the result of docetaxel inhibition rate was the opposite with a higher inhibition rate in the high expression group (14.8 vs. $26.4 \%$, low expression vs. high expression group; $\mathrm{P}=0.002$; Fig. $2 \mathrm{C}$ ). The tissues with low TS mRNA expression levels had a higher sensitivity to fluorouracil compared with those with high expression levels (21.9 vs. 14.1\%; $\mathrm{P}=0.044$; Fig. $2 \mathrm{H}$ ). The group with high TOP1 mRNA expression levels was more sensitive to irinotecan compared with those with low expression levels (18.0 vs. 28.4\%; P=0.031; Fig. 2I). However, statistically insignificant associations between genes levels and inhibition rates were also observed as follows: ERCC1 with cisplatin (38.2 vs. $48.0 \%, \mathrm{P}=0.120$; Fig. $2 \mathrm{~A}$ ), TUBB3 with docetaxel (21.5 vs. $13.3 \%, \mathrm{P}=0.052$; Fig. 2D), FBW7 with docetaxel (19.4 vs. $18.2 \%, \mathrm{P}=0.784$; Fig. $2 \mathrm{E}$ ), RRM1 with gemcitabine (20.3 vs. $25.2 \%, P=0.268$; Fig. $2 \mathrm{~F}$ ) and MDM2 with etoposide (19.7 vs. 20.4\%, $\mathrm{P}=0.670$; Fig. $2 \mathrm{G}$ ).

Association between BRCAI expression and clinical outcomes in patients treated with chemotherapy. In order to verify whether the predictive effects of screened biomarkers in personalized chemotherapy is consistent with in vitro chemosensitivity, the authors of the present study further investigated the associations of BRCA1 mRNA expression with clinical outcomes in patients with advanced esophageal cancer, who were treated with cisplatin-fluorouracil or docetaxel-fluorouracil chemotherapy. The findings indicated that patients treated with cisplatin-fluorouracil chemotherapy with low BRCA1 expression had increased mOS (16.5 vs. 6.5 months; $\mathrm{P}<0.001$; Fig. 3A, Table V) and RR (38.1 vs. 7.7\%; $\mathrm{P}=0.002$; Table V) compared with those with high expression. However, patients treated with docetaxel-fluorouracil chemotherapy with high BRCA1 mRNA expression had increased mOS (22.0 vs. 6.8 months; $\mathrm{P}<0.001$; Fig. 3B, Table V) and RR 
(57.1 vs. $20.8 \%$; $\mathrm{P}=0.023$; Table $\mathrm{V}$ ) compared with those with low BRCA1 expression.

\section{Discussion}

Treatment options in esophageal cancer have advanced over the last several years with the introduction of effective chemotherapeutics. However, personalized therapy is far from being implemented due to the lack of effective predictive biomarkers (25). Currently, the prediction of response to chemotherapy at the molecular level is primarily based on data derived from in vitro experiments (26-28). Furthermore, studies, which utilize cell culture model of tumors, organoid cultures or xenografts currently best mimic the characteristics of an in vivo tumor (29). An in vitro histoculture system is able to maintain the structure of the three-dimensional tissue and the natural tumor environment $(18,19)$. Despite mouse xenograft models having the advantages of being able to mimic the micro-environmental conditions, tumor architecture, angiogenesis and metastasis present in a real patient, the in vitro histoculture system has relative advantages of good availability, low cost, ease of handling and short intervention time. Therefore, in the present study the HDRA histoculture system was selected to determine chemosensitivity. In addition, 8 parallel culture wells were designed to test the chemosensitivity of different parts of tumor specimen to avoid the issue of tumor heterogeneity.

The efficacy rate for an individual agent using HDRA in vitro has a considerable good correlation with clinical response rate to each agent $(30,31)$. Previous studies have reported that TS and DPD expression are correlated with fluorouracil sensitivity (32-35). It was also reported that ERCC1 expression and SULF2 methylation are correlated with platinum sensitivity $(33,36)$. Furthermore, CXCR4 and TUBB3 expression are correlated with docetaxel sensitivity $(37,38)$. Aprataxin (APTX) expression also has been correlated with irinotecan sensitivity $(29,33)$, and MET expression has been correlated with crizotinib sensitivity (39) using HDRA. These results suggest that HDRA can be used in predictive biomarker studies. In addition, the qPCR method, which may be more clinically useful with the benefits of being able to provide more quantitative and accurate measurement compared with fluorescence in situ hybridization and immunohistochemistry, has been widely employed to detect the expression levels of candidate genes in the aforementioned studies. Therefore, in the present study, a total of 8 candidate biomarkers were selected based on literature review, and the gene expression patterns were analyzed using qPCR. A number of potential biomarkers for chemotherapy in esophageal cancer were identified, including BRCA1 for docetaxel or cisplatin, TS for fluorouracil and TOP1 for irinotecan. The authors then considered whether combining HDRA with qPCR for biomarker discovery may provide novel opportunities for prediction of individual response to chemotherapy. Despite the identification of a number of candidate biomarkers for esophageal cancer in the present study, whether the predictive functions of these genes may be reproduced in clinical practice still requires validation.

Previous studies have evaluated the predictive value of potential biomarkers generated from HDRA in xenograft model and in clinical settings. Shen et al (29) established different mice models with patient-derived gastric cancer xenografts and demonstrated that tumor growth is significantly suppressed in the cohort with sensitive-signature based on the expression of APTX, BRCA1 and TOP1. Yang et al (39) have reported that patient-derived tumor xenograft models with higher MET expression exhibited high sensitivity to crizotinib, and tumor shrinkage was observed in a patient with advanced gastric cancer and MET overexpression following crizotinib treatment. However, differences in metabolism, body size and genetic background between the host species and humans may have an impact on the predictive value of biomarkers.

Therefore, in the present study, a total of 72 patients with advanced esophageal cancer, who were treated with cisplatin-fluorouracil or docetaxel-fluorouracil chemotherapy, were recruited, and the predictive function of BRCA1 in personalized treatment was analyzed. Fluorouracil was used in both regimens, as the presence of BRCA1 did not confer resistance or sensitivity to fluorouracil.

High expression of BRCA1 mRNA was negatively associated with RR and mOS in patients treated with cisplatin-fluorouracil chemotherapy. Conversely, high BRCA1 expression was also positively associated with clinical outcomes in those who received docetaxel-fluorouracil chemotherapy. As a dual predictive biomarker, the results were consistent with previous findings by the present authors $(40,41)$. The findings supported the hypothesis that the use of a combination of HDRA and qPCR is able to effectively distinguish biomarkers in their ability to evaluate response to chemotherapy.

In summary, the present study observed that the level of BRCA1, TS and TOP1 mRNA present make these genes suitable as predictable biomarkers to assess the response of cisplatin, docetaxel, fluorouracil or irinotecan in patients with esophageal cancer. Furthermore, the combination of HDRA and qPCR may be an effective method for screening biomarkers to assess chemosensitivity in personalized chemotherapy for esophageal cancer.

\section{Acknowledgements}

The present study was supported by the National Natural Science Foundation of China (grant no. 81572421), the Jiangsu Provincial Commission of Health and Family Planning Program (grant no. H201555) and Huai'an Governmental Science Developing program (grant no. HACZ2014002).

\section{References}

1. Zhang HZ, Jin GF and Shen HB: Epidemiologic differences in esophageal cancer between Asian and Western populations. Chin J Cancer 31: 281-286, 2012.

2. Ferlay J, Soerjomataram I, Dikshit R, Eser S, Mathers C, Rebelo M, Parkin DM, Forman D and Bray F: Cancer incidence and mortality worldwide: Sources, methods and major patterns in GLOBOCAN 2012. Int J Cancer 136: E359-E386, 2015.

3. Ezdinli EZ, Gelber R, Desai DV, Falkson G, Moertel CG and Hahn RG: Chemotherapy of advanced esophageal carcinoma: Eastern Cooperative Oncology Group experience. Cancer 46: 2149-2153, 1980.

4. Ajani JA, Ilson DH, Daugherty K, Pazdur R, Lynch PM and Kelsen DP: Activity of taxol in patients with squamous cell carcinoma and adenocarcinoma of the esophagus. J Natl Cancer Inst 86: 1086-1091, 1994. 
5. Millar J, Scullin P, Morrison A, McClory B, Wall L, Cameron D, Philips H, Price A, Dunlop D and Eatock M: Phase II study of gemcitabine and cisplatin in locally advanced/metastatic oesophageal cancer. Br J Cancer 93: 1112-1116, 2005.

6. Denison TA and Bae YH: Tumor heterogeneity and its implication for drug delivery. J Control Release 164: 187-191, 2012.

7. O'Donnell PH and Dolan ME: Cancer pharmacoethnicity: Ethnic differences in susceptibility to the effects of chemotherapy. Clin Cancer Res 15: 4806-4814, 2009.

8. Papadaki C, Sfakianaki M, Ioannidis G, Lagoudaki E, Trypaki M, Tryfonidis K, Mavroudis D, Stathopoulos E, Georgoulias V and Souglakos J: ERCC1 and BRAC1 mRNA expression levels in the primary tumor could predict the effectiveness of the second-line cisplatin-based chemotherapy in pretreated patients with metastatic non-small cell lung cancer. J Thorac Oncol 7: 663-671, 2012.

9. Wei J, Costa C, Ding Y, Zou Z, Yu L, Sanchez JJ, Qian X, Chen H, Gimenez-Capitan A, Meng F, et al: mRNA expression of BRCA1, PIAS1, and PIAS4 and survival after second-line docetaxel in advanced gastric cancer. J Natl Cancer Inst 103: 1552-1556, 2011.

10. Wertz IE, Kusam S, Lam C, Okamoto T, Sandoval W, Anderson DJ, Helgason E, Ernst JA, Eby M, Liu J, et al: Sensitivity to antitubulin chemotherapeutics is regulated by MCL1 and FBW7. Nature 471: 110-114, 2011.

11. Urano N, Fujiwara Y, Doki Y, Kim SJ, Miyoshi Y, Noguchi S, Miyata $\mathrm{H}$, Takiguchi S, Yasuda T, Yano $\mathrm{M}$ and Monden $\mathrm{M}$ : Clinical significance of class III beta-tubulin expression and its predictive value for resistance to docetaxel-based chemotherapy in gastric cancer. Int J Oncol 28: 375-381, 2006.

12. Soong R, Shah N, Salto-Tellez M, Tai BC, Soo RA, Han HC, Ng SS, Tan WL, Zeps N, Joseph D, et al: Prognostic significance of thymidylate synthase, dihydropyrimidine dehydrogenase and thymidine phosphorylase protein expression in colorectal cancer patients treated with or without 5-fluorouracil-based chemotherapy. Ann Oncol 19: 915-919, 2008.

13. Souglakos J, Boukovinas I, Taron M, Mendez P, Mavroudis D, Tripaki M, Hatzidaki D, Koutsopoulos A, Stathopoulos E, Georgoulias V and Rosell R: Ribonucleotide reductase subunits M1 and M2 mRNA expression levels and clinical outcome of lung adenocarcinoma patients treated with docetaxel/gemcitabine. $\mathrm{Br}$ J Cancer 98: 1710-1715, 2008.

14. Rodriguez-Lopez AM, Xenaki D, Eden TO, Hickman JA and Chresta CM: MDM2 mediated nuclear exclusion of p53 attenuates etoposide-induced apoptosis in neuroblastoma cells. Mol Pharmacol 59: 135-143, 2001.

15. Tsurutani J, Nitta T, Hirashima T, Komiya T, Uejima H, Tada H, Syunichi N, Tohda A, Fukuoka M and Nakagawa K: Point mutations in the topoisomerase I gene in patients with non-small cell lung cancer treated with irinotecan. Lung Cancer 35: 299-304, 2002.

16. Kim M, Keam B, Kim TM, Kim HG, Kim JS, Lee SS, Shin SH, Kim MK, Park KU, Kim DW, et al: Phase II study of irinotecan and cisplatin combination chemotherapy in metastatic, unresectable esophageal cancer. Cancer Res Treat 49: 416-422, 2017.

17. Harstrick A, Bokemeyer C, Preusser P, Köhne-Wömpner CH, Meyer HJ, Stahl M, Knipp H, Schmoll HJ and Wilke H: Phase II study of single-agent etoposide in patients with metastatic squamous-cell carcinoma of the esophagus. Cancer Chemother Pharmacol 29: 321-322, 1992.

18. Furukawa T, Kubota T, Tanino H, Oura S, Yuasa S, Murate H, Morita K, Kozakai K, YanoT and Hoffman RM: Chemosensitivity of breast cancer lymph node metastasis compared to the primary tumor from individual patients tested in the histoculture drug response assay. Anticancer Res 20: 3657-3658, 2000.

19. Hoffman RM: To do tissue culture in two or three dimensions? That is the question. Stem Cells 11: 105-111, 1993.

20. Tanino H, Oura S, Hoffman RM, Kubota T, Furukawa T, Arimoto J, Yoshimasu T, Hirai I, Bessho T, Suzuma T, et al Acquisition of multidrug resistance in recurrent breast cancer demonstrated by the histoculture drug response assay. Anticancer Res 21: 4083-4086, 2001.

21. Kim R,Emi M, Tanabe K, Uchida Y and Toge T: Chemosensitivity testing for gastrointestinal cancer: Survival benefit potential and limitations. Anticancer Drugs 14: 715-723, 2003

22. Lee SW, Kim YM, Kim MB, Kim DY, Kim JH, Nam JH and Kim YT: In vitro chemosensitivity using the histoculture drug response assay in human epithelial ovarian cancer. Acta Med Okayama 66: 271-277, 2012.
23. Rosen MA and Sullivan D: Optimal lesion number for evaluation of tumor response in response evaluation criteria in solid tumors. J Clin Oncol 28: e159-e161, 2010.

24. Goense L, van Rossum PS, Kandioler D, Ruurda JP, Goh KL, Luyer MD, Krasna MJ and van Hillegersberg R: Stage-directed individualized therapy in esophageal cancer. Ann N Y Acad Sci 1381: 50-65, 2016.

25. Liu Y,Xiong Z, Beasley A, D'Amico T and Chen XL: Personalized and targeted therapy of esophageal squamous cell carcinoma: An update. Ann N Y Acad Sci 1381: 66-73, 2016.

26. Furukawa T, Kubota T, Watanabe M, Takahara T, Yamaguchi H, Takeuchi T, Kase S, Kodaira S, Ishibiki K, Kitajima M, et al: High in vitro-in vivo correlation of drug response using sponge-gel-supported three-dimensional histoculture and the MTT end point. Int J Cancer 51: 489-498, 1992.

27. Sevin BU, Peng ZL, Perras JP, Ganjei P, Penalver M and Averette HE: Application of an ATP-bioluminescence assay in human tumor chemosensitivity testing. Gynecol Oncol 31: 191-204, 1988

28. Bogden AE, Cobb WR, Lepage DJ, Haskell PM, Gulkin TA, Ward A, Kelton DE and Esber HJ: Chemotherapy responsiveness of human tumors as first transplant generation xenografts in the normal mouse: Six-day subrenal capsule assay. Cancer 48: 10-20, 1981.

29. Shen J, Wei J, Wang H, Yue G, Yu L, Yang Y, Xie L, Zou Z, Qian X, Ding Y, et al: A three-gene signature as potential predictive biomarker for irinotecan sensitivity in gastric cancer. J Transl Med 11: 73, 2013.

30. Furukawa T, Kubota T and Hoffman RM: Clinical applications of the histoculture drug response assay. Clin Cancer Res 1: 305-311, 1995.

31. Yoshimasu T, Oura S, Hirai I, Tamaki T, Kokawa Y, Hata K, Ohta F, Nakamura R, Kawago M, Tanino H, et al: Data acquisition for the histoculture drug response assay in lung cancer. J Thorac Cardiovasc Surg 133: 303-308, 2007.

32. Zhang Q, Shen J, Wang H, Hu J, Yu L, Xie L, Wei J, Liu B, Guan W and Qian X: TS mRNA levels can predict pemetrexed and raltitrexed sensitivity in colorectal cancer. Cancer Chemother Pharmacol 73: 325-333, 2014

33. Yue G, Wei J, Qian X, Yu L, Zou Z, Guan W, Wang H, Shen J and Liu B: Synergistic anticancer effects of polyphyllin I and evodiamine on freshly-removed human gastric tumors. PLoS One 8: e65164, 2013.

34. Kinoshita M, Kodera Y, Hibi K, Nakayama G, Inoue T, Ohashi N, Ito Y, Koike M, Fujiwara M and Nakao A: Gene expression profile of 5-fluorouracil metabolic enzymes in primary colorectal cancer: Potential as predictive parameters for response to fluorouracil-based chemotherapy. Anticancer Res 27: 851-856, 2007.

35. Fakhrejahani E, Miyamoto A and Tanigawa N: Correlation between thymidylate synthase and dihydropyrimidine dehydrogenase mRNA level and in vitro chemosensitivity to 5-fluorouracil, in relation to differentiation in gastric cancer. Cancer Chemother Pharmacol 60: 437-446, 2007.

36. Shen J, Wei J, Wang H, Yang Y, Yue G, Wang L, Yu L, Xie L, Sun X, Bian X, et al: SULF2 methylation is associated with in vitro cisplatin sensitivity and clinical efficacy for gastric cancer patients treated with a modified FOLFOX regimen. PLoS One 8: e75564, 2013

37. Ohashi T, Yoshimasu T, Oura S, Kokawa Y, Kawago M, Hirai Y, Miyasaka M, Aoishi Y, Kiyoi M, Nishiguchi H, et al: Class III beta-tubulin expression in non-small cell lung cancer: A predictive factor for paclitaxel response. Anticancer Res 35: 2669-2674, 2015.

38. Xie L, Wei J, Qian X, Chen G, Yu L, Ding Y and Liu B: CXCR4, a potential predictive marker for docetaxel sensitivity in gastric cancer. Anticancer Res 30: 2209-2216, 2010.

39. Yang Y, Wu N, Shen J, Teixido C, Sun X, Lin Z, Qian X, Zou Z, Guan W, Yu L, et al: MET overexpression and amplification define a distinct molecular subgroup for targeted therapies in gastric cancer. Gastric Cancer 19: 778-788, 2016.

40. Gao Y, Zhu J, Zhang X, Wu Q, Jiang S, Liu Y, Hu Z, Liu B and Chen X: BRCA1 mRNA expression as a predictive and prognostic marker in advanced esophageal squamous cell carcinoma treated with cisplatin- or docetaxel-based chemotherapy/chemoradiotherapy. PLoS One 8: e52589, 2013.

41. Wei B, Han Q, Xu L, Zhang X, Zhu J, Wan L, Jin Y, Qian Z, Wu J, Gao Y, et al: Effects of JWA, XRCC1 and BRCA1 mRNA expression on molecular staging for personalized therapy in patients with advanced esophageal squamous cell carcinoma. BMC Cancer 15: 331, 2015 